\title{
CÁNULA NASAL DE ALTO FLUJO: REVISIÓN SISTEMÁTICA
}

HIGH FLOW NASAL CANNULA: SYSTEMATIC REVIEW

\section{Ruth ORTIZ ${ }^{1}$}

${ }^{1}$ Universidad Nacional de Asunción, Facultad de Ciencias Médicas, Carrera de Kinesiología y Fisioterapia, San Lorenzo, Paraguay

Cómo citar este artículo: Ortiz R. Cánula nasal de alto flujo: revisión sistemática. Med. clín. soc. 2019;3(2):50-57.

\section{RESUMEN}

Introducción: Hay una creciente aplicación de la terapia con oxígeno de cánula nasal de alto flujo (HFNC) como primera línea o uso complementario durante la ventilación mecánica no invasiva para evitar la intubación endotraqueal y mejorar el resultado hospitalario corto. Algunas ventajas bien conocidas como las aplicaciones fáciles, la comodidad del paciente son determinantes clave que justifican la presente investigación. Metodología: Es un estudio de revisión sistemática de la literatura médica. Se realizó una búsqueda en línea de publicaciones relevantes en PubMed, CICCO y Web of Science. La búsqueda se restringió a artículos escritos en inglés o español. Resultados: Se evaluaron 692 textos completos se los cuales finalmente se seleccionaron 13, la principal causa de eliminación fue, que iban dirigidos a población pediátrica o que no indicaban de forma explícita el mecanismo de acción y las indicaciones clínicas del uso de la cánula de alto flujo. Conclusión: Se ha propuesto que la cánula de alto flujo puede proporcionar varios beneficios. Entre estos se encuentran el mantenimiento de una $\mathrm{FiO}_{2}$ constante, la generación de una presión positiva al final de la espiración (PEEP), la reducción del espacio muerto anatómico, la mejora del aclaramiento mucociliar y la reducción del trabajo de respiración.

Palabras clave: cánula de alto flujo; kinesiología; fisioterapia.

\section{ABSTRACT}

Introduction: There is a growing application of high flow nasal cannula oxygen therapy (HFNC) as a first line or complementary use during non-invasive mechanical ventilation to avoid endotracheal intubation and improve the short hospital outcome. Some advantages well known as easy applications, patient comfort are key determinants that justify this research. Methodology: It is a systematic review study of the medical literature. An online search of relevant publications was conducted in PubMed, CICCO and Web of Science. The search was restricted to articles written in English or Spanish. Results: 692 full texts were evaluated, 13 of which were finally selected, the main cause of elimination was, that they were aimed at the pediatric population or that they did not explicitly indicate the mechanism of action and the clinical indications of the use of the cannula high flow Conclusion: It has been proposed that the high flow cannula can provide several benefits. Among these are the maintenance of a constant $\mathrm{FiO}_{2}$, the generation of a positive pressure at the end of expiration (PEEP), the reduction of anatomical dead space, the improvement of mucociliary clearance and the reduction of breathing work.

Keywords: high flow cannula; kinesiology; physiotherapy. 


\section{INTRODUCCIÓN}

En los últimos 10 años, hay una creciente aplicación de la terapia con oxígeno de cánula nasal de alto flujo (HFNC) (1-15) como primera línea o uso complementario durante la ventilación mecánica no invasiva para evitar la intubación endotraqueal y mejorar el resultado hospitalario corto. Algunas ventajas bien conocidas como las aplicaciones fáciles, la comodidad del paciente son determinantes clave que justifican la presente investigación (16).

La terapia de oxígeno es el primer paso en la prevención y el tratamiento de la insuficiencia respiratoria hipoxémica y tradicionalmente se ha administrado con puntas o mascarillas nasales. Sin embargo, los caudales máximos que estos dispositivos pueden suministrar están limitados debido a la incomodidad generada como consecuencia del calor y la humedad insuficientes proporcionados al gas administrado. Aunque la oxigenoterapia de alto flujo actualmente se define como flujos mayores de $30 \mathrm{I} / \mathrm{min}$, se acepta que los flujos de hasta $15 \mathrm{I} / \mathrm{min}$ pueden administrarse utilizando puntas o mascarillas nasales convencionales; este flujo es mucho menor que el flujo inspiratorio máximo de un paciente con disnea (17).

Además, los flujos que superan los $6 \mathrm{I} /$ min pueden provocar una humidificación insuficiente en la mucosa nasal, incluso cuando se utiliza un humidificador de burbujas frías. Por lo tanto, el aire de la habitación diluye el oxígeno suplementario, lo que resulta en una disminución significativa en la fracción del oxígeno inspirado $\left(\mathrm{FiO}_{2}\right)$ que finalmente llega a los alvéolos (17).

Durante la última década, se ha introducido el flujo alto nasal para la terapia de oxígeno en adultos, como una extensión natural de su uso en neonatos y niños. El dispositivo consiste en un mezclador de aire/oxígeno conectado a través de un humidificador calentado activo a una cánula nasal, a través de un circuito inspiratorio calentado de una sola extremidad.

Recientemente, las indicaciones para el sistema de alto flujo nasal se han expandido, aunque no existen pautas o algoritmos establecidos para guiar su uso. Por lo que la revisión hará énfasis en las implicancias clínicas en adultos y su comparación con los dispositivos de oxígeno convencionales o la ventilación no invasiva. El objetivo general de la investigación es determinar el estado del arte sobre la cánula de alto flujo.

\section{METODOLOGÍA}

Es un estudio de revisión sistemática de la literatura médica. Se realizó una búsqueda en línea de publicaciones relevantes en PubMed, CICCO y Web of Science. La búsqueda se restringió a artículos escritos en inglés o español. El periodo de búsqueda es desde 2007 al 2019. Las palabras clave de búsqueda fueron: "high flow nasal cannula", "high flow nasal therapy", "high flow nasal oxygen", "high flow oxygen therapy", "high flow therapy", "optiflow (respiration)" and "nasal highflow", luego se procedió a recuperar los resúmenes de dichos trabajos y búsqueda manual de publicaciones repetidas.

Los tipos de estudio incorporados fueron: revisión sistemática, reporte de casos, ensayo clínico, estudio comparativo, estudios de evaluación, estudios multicéntricos, estudios de casos y controles, estudios observacionales, estudios de cohorte y metaanálisis. Se excluyeron aquellos estudios que involucren población pediátrica. 
Se procedió a realizar el diagrama de flujo para la selección del estudio. Después de determinar la relevancia de cada artículo, los artículos se resumieron en forma cualitativa para cumplir el objetivo de la investigación.

\section{RESULTADOS}

\section{Descripción de los resultados}

Se realizó una revisión sistemática de la literatura con el fin de describir el estado del arte sobre el uso de la cánula de alto flujo en publicaciones médicas en las principales bases de datos. Se evaluaron 692 textos completos se los cuales finalmente se seleccionaron 13, la principal causa de eliminación fue, que iban dirigidos a población pediátrica o que no indicaban de forma explícita el mecanismo de acción y las indicaciones clínicas del uso de la cánula de alto flujo. En la figura 1 se observa el diagrama de flujo de la selección.

Figure 1. Diagrama de flujo para la selección de los artículos

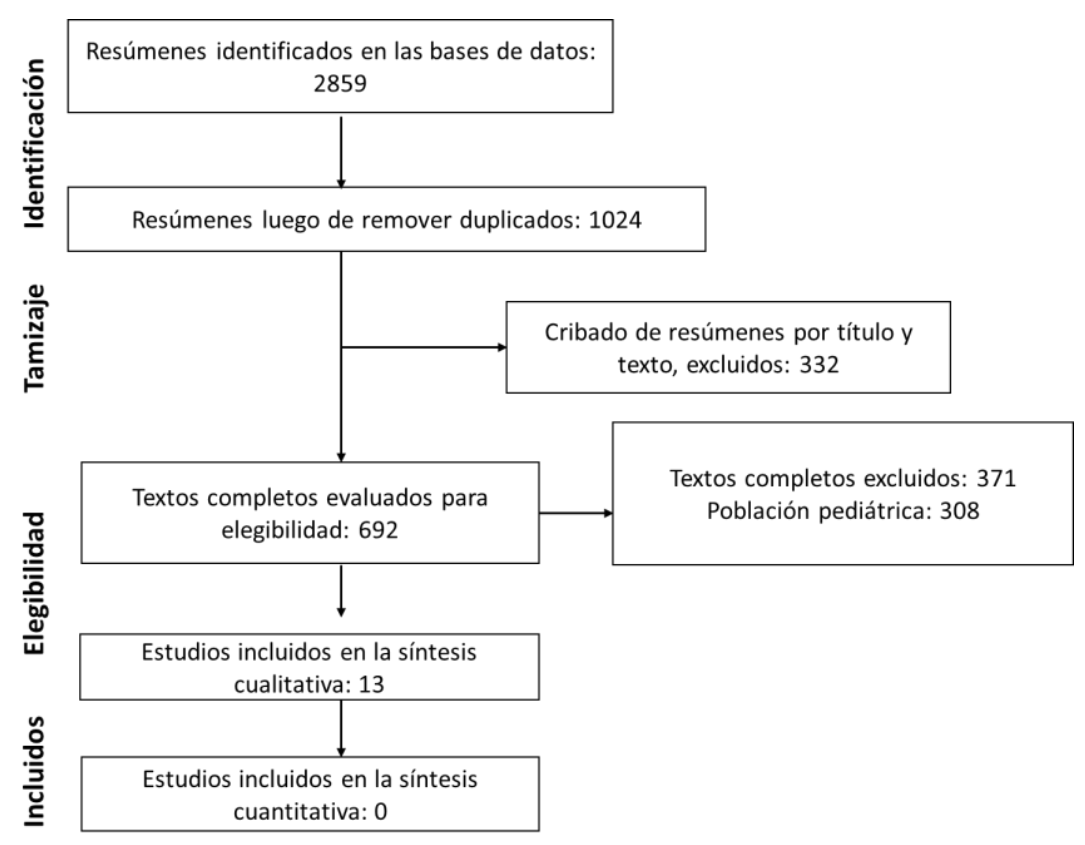

\section{Síntesis cualitativa de los hallazgos}

\section{Definición de alto flujo nasal}

La oxigenoterapia de alto flujo con cánula nasal (High Flow Nasal Cannula Oxygen Therapy [HFNC] por sus siglas en inglés) es una alternativa a la oxigenoterapia convencional, de reciente introducción. Este sistema funciona con una mezcladora de oxígeno y aire, que permite aplicar una $\mathrm{FIO}_{2}$ del 21 al 100\%, generando velocidades de flujo de hasta 60lpm (18).

La mezcla de oxígeno y aire pasa a través de un humidificador a un circuito inspiratorio calentado (para evitar la condensación). Posteriormente esta mezcla es administrada al paciente a través de cánulas nasales de gran diámetro. La característica principal de la HFNC es que permite administrar un alto flujo humidificado por encima del flujo inspiratorio máximo. Permite

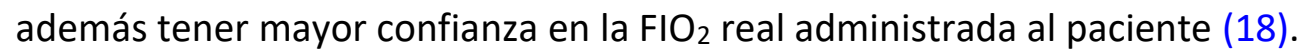


Mecanismo de acción

Se proponen dos categorías importantes de mecanismos de acción que respaldan los beneficios clínicos informados de cánula nasal de alto flujo. El primer mecanismo está relacionado con el acondicionamiento óptimo del gas suministrado porque las mezclas de aire/oxígeno nasal se calientan y se humidifican de manera cercana a las condiciones fisiológicas $(19,20)$.

Por lo tanto, el flujo de oxígeno se tolera mejor y proporciona un mayor confort respiratorio, especialmente con flujos de hasta $60 \mathrm{~L} / \mathrm{min}(19,20)$. El segundo mecanismo está relacionado con la entrega de alto flujo (> $30 \mathrm{~L} / \mathrm{min}$ ). La terapia con cánula nasal de alto flujo genera una presión positiva en la vía aérea dependiente del flujo $(20,21)$.

A $35 \mathrm{~L} / \mathrm{min}$, la presión media de las vías respiratorias medida con un catéter nasofaríngeo fue de 1,2 $\pm 0,8 \mathrm{cmH}_{2} \mathrm{O}$, boca abierta, aumentando hasta $2,7 \pm 1,0 \mathrm{cmH}_{2} \mathrm{O}$, boca cerrada y $3,3 \pm$ $1,0 \mathrm{cmH}_{2} \mathrm{O}$ a $50 \mathrm{~L} / \mathrm{min}(22,23)$.

Un estudio fisiológico demostró presiones más bajas con cánula nasal de alto flujo con el valor mediano más alto por debajo de $2,5 \mathrm{~cm} \mathrm{H}_{2} \mathrm{O}$ a $45 \mathrm{I} / \mathrm{min}$, boca cerrada (20). Esta diferencia entre los estudios podría explicarse por la medición de la presión de la vía aérea dentro de la tráquea (20), más distal del dispositivo, en lugar de en la nasofaringe (22) y la disminución de la presión de la vía aérea cuando los pacientes respiran con la boca abierta $(20,24)$.

\section{Implicancias Clínicas}

Los sistemas convencionales de oxígeno de flujo alto y bajo se utilizan tradicionalmente como tratamiento de apoyo de primera línea para el fallo agudo respiratorio (ARF) hipoxémico. Desafortunadamente, en ambos sistemas, a altas tasas de flujo inspiratorio hay una limitación del calentamiento y la humidificación del oxígeno inspirado, así como un aumento del arrastre del aire de la habitación, lo que conduce a la dilución del oxígeno y al descenso de la $\mathrm{FiO}_{2}(25)$.

Los principios y mecanismos de acción de HFNC lo convierten en un dispositivo atractivo y prometedor de suministro de oxígeno para pacientes adultos con ARF hipoxémico. Los efectos beneficiosos de la HFNC en ambos parámetros objetivos (frecuencia respiratoria y saturación arterial de oxígeno medida por oximetría de pulso $\left(\mathrm{SpO}_{2}\right)$ ) y subjetivos, como el grado de disnea detectada, se confirmaron en dos estudios en pacientes con ARF que recibieron oxígeno a través de HFNC o mascara facial.

La HFNC se asoció con menos disnea, menor frecuencia respiratoria y mayor oxigenación y comodidad en general $(26,27)$. El corto período de implementación (30 min) de HFNC $(27)$ y el inicio tardío de su uso (26) no permitieron conclusiones adicionales con respecto a la efectividad de HFNC en ARF.

Sztrymf et al. (28) estudiaron la eficacia, la seguridad y el resultado de la aplicación inmediata de HFNC en pacientes de unidades de cuidados intensivos (UCI) con ARF hipoxémico sin ninguna limitación temporal de su aplicación. El uso de HFNC se asoció con una reducción significativa de la frecuencia respiratoria, la frecuencia cardíaca, la puntuación de la disnea, la retracción supraclavicular y la asincronía toraco-abdominal y una mejora significativa en la $\mathrm{SpO}_{2}$. Estas mejoras fueron evidentes en los primeros 15 a 30 minutos después de la aplicación de HFNC y duraron todo el período de estudio sin ningún efecto secundario inesperado. 
Más del 75\% de los pacientes evitó la intubación y, por lo tanto, los autores concluyeron que el HFNC tiene un efecto favorable sobre los signos clínicos y la oxigenación en pacientes críticos con IRA (28). Estos resultados alentadores fueron confirmados por el mismo grupo de autores un año más tarde en un estudio observacional prospectivo en pacientes con IRA causada por neumonía y sepsis adquirida en la comunidad (29). Además, el HFNC puede ser más efectivo en el tratamiento de un ARF hipoxémico de leve a moderado en comparación con el oxígeno administrado a través de una máscara facial, con menos desaturaciones y menos necesidad de VNI, como se informó en un estudio prospectivo aleatorizado de Parke et al. (30).

\section{DISCUSIÓN}

Se ha propuesto que la cánula de alto flujo puede proporcionar varios beneficios. Entre estos se encuentran el mantenimiento de una $\mathrm{FiO}_{2}$ constante, la generación de una presión positiva al final de la espiración (PEEP), la reducción del espacio muerto anatómico, la mejora del aclaramiento mucociliar y la reducción del trabajo de respiración.

De la revisión se desprende que los potenciales beneficios que tiene este tipo de terapéutica son: mantenimiento de $\mathrm{FiO}_{2}$ constante, generación de una presión positiva al final de la espiración, disminución en el espacio muerto anatómico, despeje mucociliar mejorado, disminución del trabajo de respiración (31).

Dados estos potenciales beneficios, las indicaciones más usuales de la cánula de alto flujo son las siguientes: insuficiencia respiratoria hipoxémica aguda, periodo posterior a la extubación, periodo de pre-intubación, infección respiratoria, enfermedad obstructiva de las vías respiratorias y el uso en emergencias (32). Los problemas de presentación más comunes en pacientes que acuden al servicio de urgencias son disnea aguda e hipoxemia, y la oxigenoterapia es un tratamiento de apoyo esencial para abordar estos síntomas. La terapia de cánula de alto flujo con calefacción y humidificación representa una nueva alternativa a la terapia de oxígeno tradicional (32).

En toda la revisión de la literatura se observó que la cánula de alto flujo se muestra prometedor para reducir la necesidad de intubación, ventilación mecánica y escalada de soporte respiratorio en comparación con la oxigenoterapia convencional, sin embargo, el número de ensayos clínicos aleatorizados no es suficiente para proporcionar evidencia sólida (33).

Las limitaciones del estudio son las intrínsecas del tipo de estudio, ya que por más que la revisión sistemática busca objetivar las búsquedas y hacerlas replicables, en contraposición de las revisiones narrativas tradicionales, tiene un componente subjetivo, el sesgo de selección, puesto que finalmente los investigadores deciden qué información incluir y cuál no, por lo que se pudo haber incurrido en dicho sesgo. Debería, por la cantidad de resultados encontrados, realizarse un metaanálisis para realizar una síntesis y un análisis cuantitativos sobre el uso de la cánula de alto flujo en pacientes adultos.

\section{CONFLICTOS DE INTERÉS Y FUENTE DE FINANCIACIÓN}

La autora declara no poseer conflictos de interés. Fuente de financiación: ninguna. 


\section{REFERENCIAS BIBLIOGRÁFICAS}

1. Wettstein RB. A fresh look at the physiologic effects of high-flow nasal cannulae and the role they play in patient care. Respir Care. 2013;58(4):715-6. https://dx.doi.org/10.4187/respcare.02388

2. Roca O, Pérez-Terán P, Masclans JR, Pérez L, Galve E, Evangelista A, et al. Patients with New York Heart Association class III heart failure may benefit with high flow nasal cannula supportive therapy: high flow nasal cannula in heart failure. J Crit Care. 2013;28(5):741-6. https://dx.doi.org/10.1016/j.jcrc.2013.02.007

3. Ritchie JE, Williams AB, Gerard C, Hockey H. Evaluation of a humidified nasal highflow oxygen system, using oxygraphy, capnography and measurement of upper airway pressures. Anaesth Intensive Care. 2011;39(6):1103-10. https://dx.doi.org/10.1177/0310057X1103900620

4. Peters SG, Holets SR, Gay PC. High-flow nasal cannula therapy in do-not-intubate patients with hypoxemic respiratory distress. Respir Care. 2013;58(4):597-600. https://dx.doi.org/10.4187/respcare.01887

5. Nilius G, Franke K-J, Domanski U, Rühle K-H, Kirkness JP, Schneider H. Effects of nasal insufflation on arterial gas exchange and breathing pattern in patients with chronic obstructive pulmonary disease and hypercapnic respiratory failure. Adv Exp Med Biol. 2013;755:27-34. https://dx.doi.org/10.1007/978-94-007-4546-9 4

6. Mosca F, Colnaghi M, Agosti M, Fumagalli M. High-flow nasal cannula: transient fashion or new method of non-invasive ventilatory assistance? J Matern-Fetal Neonatal Med Off J Eur Assoc Perinat Med Fed Asia Ocean Perinat Soc Int Soc Perinat Obstet. 2012;25 4:68-9. https://dx.doi.org/10.3109/14767058.2012.715003

7. Manley BJ, Owen LS, Doyle LW, Andersen CC, Cartwright DW, Pritchard MA, et al. High-flow nasal cannulae in very preterm infants after extubation. $N$ Engl J Med. 2013;369 (15):1425-33. https://dx.doi.org/10.1056/NEJMoa1300071

8. Haq I, Gopalakaje S, Fenton AC, McKean MC, J O'Brien C, Brodlie M. The evidence for high flow nasal cannula devices in infants. Paediatr Respir Rev. 2014;15(2):12434. https://dx.doi.org/10.1016/i.prrv.2013.12.002

9. Gotera C, Díaz Lobato S, Pinto T, Winck JC. Clinical evidence on high flow oxygen therapy and active humidification in adults. Rev Port Pneumol. 2013;19(5):217-27. https://dx.doi.org/10.1016/i.rppneu.2013.03.005

10. Frizzola M, Miller TL, Rodriguez ME, Zhu Y, Rojas J, Hesek A, et al. High-flow nasal cannula: impact on oxygenation and ventilation in an acute lung injury model. Pediatr Pulmonol. 2011;46(1):67-74. https://dx.doi.org/10.1002/ppul.21326

11. Fernandez-Alvarez JR, Gandhi RS, Amess P, Mahoney L, Watkins R, Rabe H. Heated humidified high-flow nasal cannula versus low-flow nasal cannula as weaning mode from nasal CPAP in infants $\leq 28$ weeks of gestation. Eur J Pediatr. 2014;173(1):93-8. https://dx.doi.org/10.1007/s00431-013-2116-2

12. Esquinas AM, Nakagawa NK, Da Silva LFF. Comment on "humidification with highflow nasal cannula and airway epithelial cells: caution, still learning from an extremely complex environment." Pulm Med. 2012;2012:368713. https://dx.doi.org/10.1155/2012/368713 
13. Esquinas AM, Martin C. High-flow nasal cannula oxygen therapy in the emergency department: welcome, but selection should be the first step. Respir Care. 2013;58(5):e66-67. https://dx.doi.org/10.4187/respcare.02286

14. Collins CL, Barfield C, Horne RSC, Davis PG. A comparison of nasal trauma in preterm infants extubated to either heated humidified high-flow nasal cannulae or nasal continuous positive airway pressure. Eur J Pediatr. 2014;173(2):181-6. https://dx.doi.org/10.1007/s00431-013-2139-8

15. Chang GY, Cox CA, Shaffer TH. Nasal cannula, CPAP, and high-flow nasal cannula: effect of flow on temperature, humidity, pressure, and resistance. Biomed Instrum Technol .2011;45(1):69-74. https://dx.doi.org/10.2345/0899-8205-45.1.69

16. Esquinas AM, Karim HMR, Soo Hoo GW. Insight to the growing utilizations of high flow nasal oxygen therapy over non-invasive ventilation in community teaching hospital: alternative or complementary? Hosp Pract. 2018;46(4):170-1. https://dx.doi.org/10.1080/21548331.2018.1510283

17. Hernández G, Roca O, Colinas L. High-flow nasal cannula support therapy: new insights and improving performance. Crit Care. 2017;21(1):62. https://dx.doi.org/10.1186/s13054-017-1640-2

18. Demelo-Rodríguez P, Olmedo Samperio M, Gaitán Tocora DG, Cano Ballesteros JC, Andueza Lillo JA. Oxigenoterapia de alto flujo con cánula nasal: estudio preliminar en pacientes hospitalizados. Arch Bronconeumol. 2015;51(12):657-9. https://dx.doi.org/10.1016/j.arbres.2015.03.015

19. Chanques G, Constantin J-M, Sauter M, Jung B, Sebbane M, Verzilli D, et al. Discomfort associated with underhumidified high-flow oxygen therapy in critically ill patients. Intensive Care Med. 2009;35(6):996-1003. https://dx.doi.org/10.1007/s00134-009-1456-x

20. Chanques G, Riboulet F, Molinari N, Carr J, Jung B, Prades A, et al. Comparison of three high flow oxygen therapy delivery devices: a clinical physiological cross-over study. Minerva Anestesiol. 2013;79(12):1344-55. URL.

21. Mündel T, Feng S, Tatkov S, Schneider H. Mechanisms of nasal high flow on ventilation during wakefulness and sleep. J Appl Physiol. 2013;114(8):1058-65. https://dx.doi.org/10.1152/japplphysiol.01308.2012

22. Parke RL, Eccleston ML, McGuinness SP. The effects of flow on airway pressure during nasal high-flow oxygen therapy. Respir Care. 2011;56(8):1151-5. https://dx.doi.org/10.4187/respcare.01106

23. Parke RL, McGuinness SP. Pressures delivered by nasal high flow oxygen during all phases of the respiratory cycle. Respir Care. 2013;58(10):1621-4. https://dx.doi.org/10.4187/respcare.02358

24. Groves N, Tobin A. High flow nasal oxygen generates positive airway pressure in adult volunteers. Aust Crit Care Off J Confed Aust Crit Care Nurses. 2007;20(4):12631. https://dx.doi.org/10.1016/j.aucc.2007.08.001

25. O'Driscoll BR, Howard LS, Davison AG. BTS guideline for emergency oxygen use in adult patients. Thorax. 2008;63(Suppl 6):vi1-68. https://dx.doi.org/10.1136/thx.2008.102947

26. Lenglet H, Sztrymf B, Leroy C, Brun P, Dreyfuss D, Ricard J-D. Humidified High Flow Nasal Oxygen During Respiratory Failure in the Emergency Department: Feasibility and Efficacy. Respir Care. 2012;57(11):1873-8. https://dx.doi.org/10.4187/respcare.01575 
27. Roca O, Riera J, Torres F, Masclans JR. High-Flow Oxygen Therapy in Acute Respiratory Failure. Respir Care. 2010;55(4):408-13. URL.

28. Sztrymf B, Messika J, Bertrand F, Hurel D, Leon R, Dreyfuss D, et al. Beneficial effects of humidified high flow nasal oxygen in critical care patients: a prospective pilot study. Intensive Care Med. 2011;37(11):1780-6. https://dx.doi.org/10.1007/s00134-011-2354-6

29. Sztrymf B, Messika J, Mayot T, Lenglet H, Dreyfuss D, Ricard J-D. Impact of highflow nasal cannula oxygen therapy on intensive care unit patients with acute respiratory failure: a prospective observational study. J Crit Care. 2012;27(3):324.e9-13. https://dx.doi.org/10.1016/i.jcrc.2011.07.075

30. Parke RL, McGuinness SP, Eccleston ML. A Preliminary Randomized Controlled Trial to Assess Effectiveness of Nasal High-Flow Oxygen in Intensive Care Patients. Respir Care. 2011;56(3):265-70. https://dx.doi.org/10.4187/respcare.00801

31. Helviz Y, Einav S. A Systematic Review of the High-flow Nasal Cannula for Adult Patients. Crit Care. 2018;22(1):71. https://dx.doi.org/10.1186/s13054-018-1990-4

32. Zhang J, Lin L, Pan K, Zhou J, Huang X. High-flow nasal cannula therapy for adult patients. J Int Med Res. 2016;44(6):1200-11. https://dx.doi.org/10.1177/0300060516664621

33. Zhao H, Wang H, Sun F, Lyu S, An Y. High-flow nasal cannula oxygen therapy is superior to conventional oxygen therapy but not to noninvasive mechanical ventilation on intubation rate: a systematic review and meta-analysis. Crit Care Lond Engl. 2017;21(1):184. https://dx.doi.org/10.1186/s13054-017-1760-8 